\title{
A Case of Streptococcus pyogenes Sepsis of Possible Oral Origin
}

Scott Trapp, DDS; Wayne Scott, DDS

\section{The importance of integrating the dental service in overall case management is highlighted in this case of infection.}

Editor's Note: This article has been adapted from an article originally published in Federal Practitioner (Trapp S, Scott W. A case of Streptococcus pyogenes sepsis of possible oral origin. Fed Prac. 2017;34[10]:3132. http://www.fedprac.com).

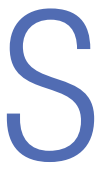

epsis can be the result of single or multiple factors and sources of infection. Oral sources of sepsis and systemic infection are not commonly considered as the first potential source of infection when evaluating a septic patient. Oral infections of odontogenic or periodontal origin are frequently associated with localized or diffuse cellulitis of the head and neck region. ${ }^{1}$ The patient's health status and complicating problems, such as an immunocompromising condition, can further reduce the immune response for controlling chronic sources of infection. This in turn can lead to acute manifestations such as cellulitis, sepsis, or necrotizing fasciitis. Necrotizing fasciitis is caused by a polymicrobial or mixed aerobic-anaerobic infection from a variety of sources, including Streptococcus pyogenes.

\section{Case}

A 57-year-old woman with a history of major depressive disorder, paroxysmal atrial fibrillation, and opioid dependence that was in remission for more than 3 years was brought to the ED by a family member after the patient developed confusion and

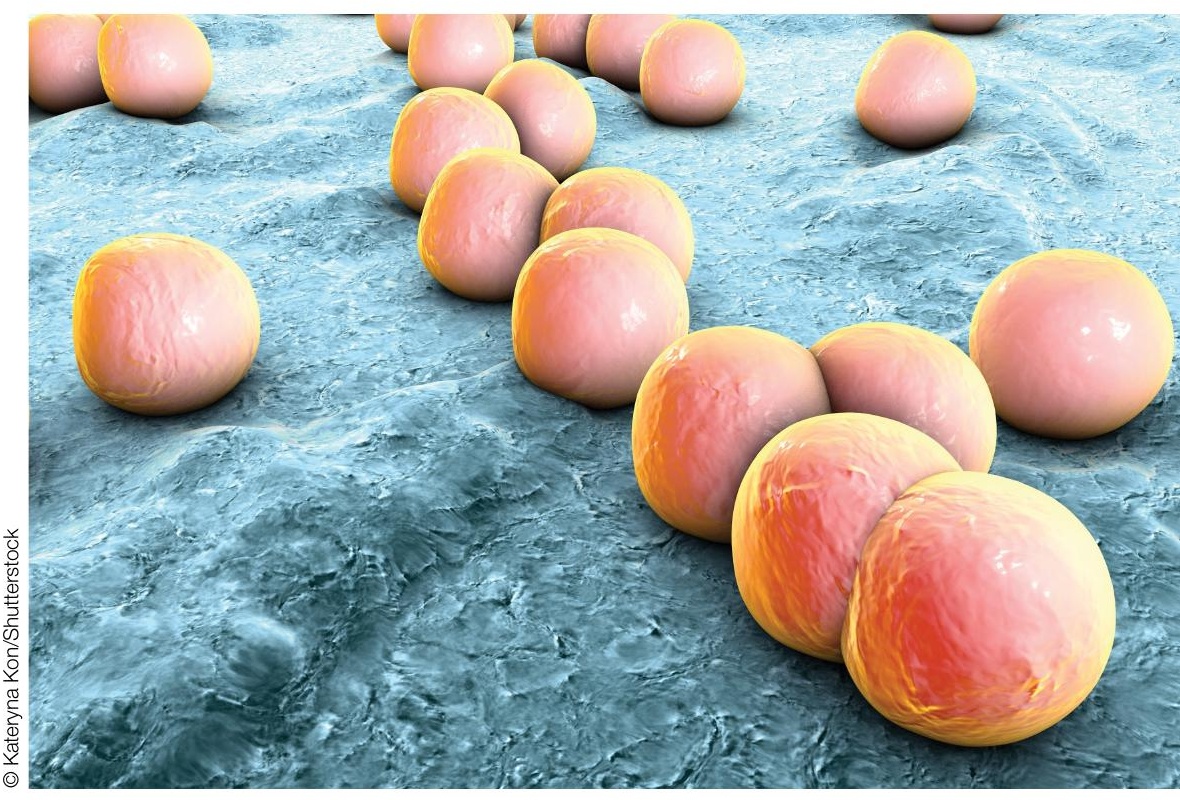

Dr Trapp the Chief, Dental Service, Veterans Health Administration St. Louis Health Care System, St. Louis, Missouri. Dr Scott is a maxillofacial surgeon, VA St. Louis Health Care System, St. Louis, Missouri.

Authors' Disclosure Statement: The authors report no actual or potential conflict of interest in relation to this article.

Disclaimer: The opinions expressed herein are those of the authors and do not necessarily reflect those of Federal Practitioner, Frontline Medical Communications Inc, the US Government, or any of its agencies.

DOI: 10.12788/emed.2017.0068 
lethargy. She was primarily experiencing right breast pain and swelling. The breast pain was associated with high fevers, nausea, vomiting, and chills.

On examination, the patient's vital signs were: blood pressure (BP), 109/58 mm Hg; heart rate, 160 beats/min; respiratory rate, 22 breaths/min; and temperature, $104^{\circ} \mathrm{F}$. Laboratory evaluation was significant for a white blood cell count (WBC) of $8.7 \times 10^{3}$. There was a noted skin abrasion on the patient's right hand. She was lethargic and confused. Blood cultures were positive for $S$ pyogenes, and a swab of the right breast was negative for bacterial growth.

The patient was admitted to the medical intensive care unit (MICU) and placed on two vasopressors for control of low BP and assistance with low urine output. After a 6-L fluid resuscitation, the patient was started on vancomycin and piperacillin/ tazobactam for possible cellulitis causing sepsis. An echocardiogram was negative for endocarditis. The patient continued to decline the following day with continuing tachycardia and tachypnea with hypotension and was intubated. Pulmonology services was consulted for possible acute respiratory distress syndrome secondary to sepsis; general surgery services was consulted for possible necrotizing fasciitis of the chest wall; and cardiology services was consulted for low-cardiac output.

On hospital day 4, the patient was taken to surgery for exploration, drainage, and debridement of the right axilla and breast; cultures were taken, and lack of organism growth was noted. While in the MICU, she was followed by the infectious disease service as her WBC remained elevated and peaking at $32.6 \times 10^{3}$, while blood cultures were negative for bacterial growth.

The dental service was consulted on hospital day 5 to evaluate for other possible sources of infection. Upon examination, the patient's oral condition was noted as having advanced chronic periodontal disease that required full-mouth extraction. The patient remained hemodynami- cally unstable with platelet counts below $50 \times 10^{9} / \mathrm{L}$ until hospital day 7 , at which time she was taken for surgery for full mouth extraction and associated alveoloplasty. Upon extraction, the patient continued to improve and was extubated on day 11 with platelets and WBC returning to normal levels by day 13 of her hospital stay. The patient remained hospitalized for a total MICU stay of 20 days and rehabilitation stay of more than 2 weeks.

\section{Discussion}

Oral infections most often present with acute onset and noted oral-facial cellulitis or abscess. Oral source of septicemia often are considered after ruling out most other potential sources. Although it is not certain that this case is directly related to the advanced chronic periodontal disease, $S$ pyogenes has been noted to be a pathogen in periodontal disease progression.

According to the American Dental Association in 2012, dental visits to the ED cost the US health care system $\$ 1.6$ billion and an average cost of $\$ 749$ per visit. There are more than 2 million ED visits each year for dental pain and infection, and 39\% return due to nonresolution of the dental problem. Patients return to the ED due to lack of access and resources to routine and emergent dental care. ${ }^{2}$ The average daily cost of an MICU stay with mechanical ventilation was $\$ 2,193$ in 2002. This particular case consisted of 11 days of mechanical ventilation, 20 MICU days, and an additional 20 days of inpatient rehabilitation which resulted in costs that exceeded $\$ 50,000 .^{3}$

\section{References}

1. Krishnan V, Johnson JV, Helfric JF. Management of maxillofacial infections: a review of 50 cases. J Oral Maxillofac Surg. 1993;51(8):868-873; discussion 873874.

2. Wall T, Vujicic M. Emergency department use for dental conditions continues to increase. American Dental Association: Health Policy Institute. http:// www.ada.org/ /media/ADA/Science $\% 20$ and $\% 20$ Research/HPI/Files/HPIBrief_0415_2.ashx. Published April 2015. Accessed November 6, 2017.

3. Dasta JF, McLaughlin TP, Mody SH, Piech CT. Daily cost of an intensive care unit day: the contribution of mechanical ventilation. Crit Care Med. 2005;33(6):1266-1271. 